\title{
People with Motor Disabilities Using Gaze to Control Telerobots
}

\section{Zhang, Guangtao; Hansen, John Paulin}

\section{Published in:}

Extended Abstracts of the $2020 \mathrm{CHI}$ Conference on Human Factors in Computing Systems

Link to article, DOI:

$10.1145 / 3334480.3382939$

Publication date:

2020

Document Version

Publisher's PDF, also known as Version of record

Link back to DTU Orbit

Citation $(A P A)$ :

Zhang, G., \& Hansen, J. P. (2020). People with Motor Disabilities Using Gaze to Control Telerobots. In Extended Abstracts of the $2020 \mathrm{CHI}$ Conference on Human Factors in Computing Systems Association for Computing Machinery. https://doi.org/10.1145/3334480.3382939

\section{General rights}

Copyright and moral rights for the publications made accessible in the public portal are retained by the authors and/or other copyright owners and it is a condition of accessing publications that users recognise and abide by the legal requirements associated with these rights.

- Users may download and print one copy of any publication from the public portal for the purpose of private study or research.

- You may not further distribute the material or use it for any profit-making activity or commercial gain

- You may freely distribute the URL identifying the publication in the public portal 


\section{People with Motor Disabilities Using Gaze to Control Telerobots}

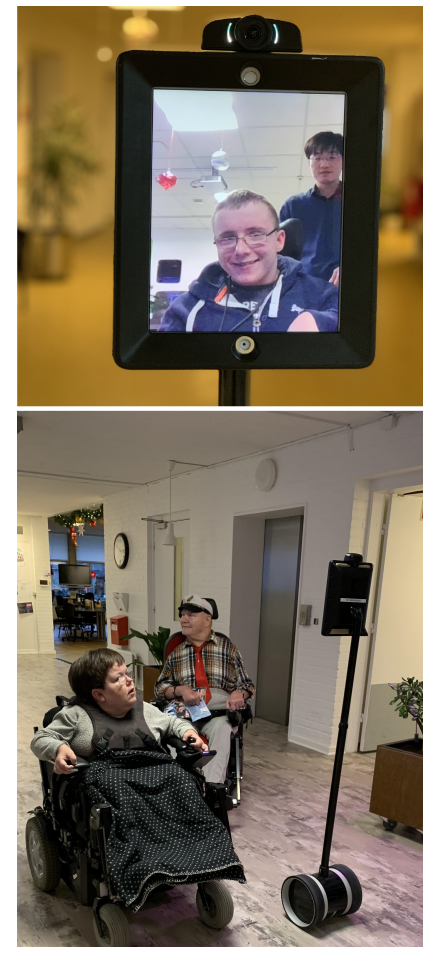

Figure 1: One of the participants communicates with his friends via a telepresence robot.

\section{Guangtao Zhang}

Technical University of Denmark

Kgs. Lyngby, Denmark

guazha@dtu.dk

John Paulin Hansen

Technical University of Denmark

Kgs. Lyngby, Denmark

jpha@dtu.dk

Permission to make digital or hard copies of part or all of this work for personal or classroom use is granted without fee provided that copies are not made or distributed for profit or commercial advantage and that copies bear this notice and the full citation on the first page. Copyrights for third-party components of this work must be honored. For all other uses, contact the owner/author(s).

CHI '20 Extended Abstracts, April 25-30, 2020, Honolulu, HI, USA.

(c) 2020 Copyright is held by the author/owner(s).

ACM ISBN 978-1-4503-6819-3/20/04.

http://dx.doi.org/10.1145/3334480.3382939

\begin{abstract}
Telerobots may give people with motor disabilities access to education, events and places. Eye-gaze interaction with these robots is an option when hands are not functional. Gaze control of telerobots has not yet been evaluated by people from this target group. We conducted a field study with five users in a care-home to investigate their preferences and challenges when driving telerobots via our gazecontrolled robotic telepresence system. We used a Wizard of $\mathrm{Oz}$ method to explore gaze and speech interaction, and experience prototyping to consider robot designs and types of displays (e.i. monitors versus head-mounted displays)
\end{abstract}

\section{Author Keywords}

Human-robot interaction, gaze interaction, telepresence, accessibility, motor disabilities, assistive technology, inclusion, robot-mediated communication, navigation, headmounted displays, experience prototyping, Wizard of Oz.

\section{CCS Concepts}

-Human-centered computing $\rightarrow$ Accessibility; Accessibility systems and tools:

\section{Introduction}

Telerobots have several potential usages for people with motor disabilities (MD) [26]. Recent development of telepresence robots [11] may provide people with MD a sense of 


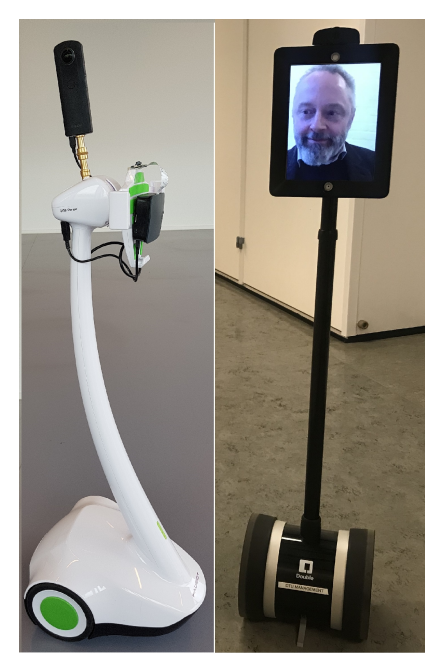

Figure 2: The two robots applied in the field study: a) A Padbot robot (left) that we attached with a 360 degree camera (but without a monitor showing the users face); $b$ ) A Double robot (right) with a live face video. presence within a remote environment. Several manufacturers offer wifi-based telerobots, e.g. Double Robotics and Padbot (see Fig. 2).

Robotic telepresence systems [32] have become increasingly popular for collaboration between geographically distributed teams[12], at academic conferences[23], for children-teacher communication[29], for relationships of long-distance couples [36], for seniors with mobility impairments[27], and for outdoor activities [8]. However, people with MD have been ignored when studying telerobot interaction [34]. Previous studies on assistive technology emphasized the importance of independence for people with MD, who often preferred to retain as much control authority as possible[18]. So while fully autonomous telpresence robots have been suggested in previous work, for instance automatically following a person in a remote environment [4], it is an open question as to level of autonomy people with MD would prefer.

\section{Alternative interaction with telerobots}

Keyboard and mouse or joysticks are most commonly used for telerobot systems. Alternative vehicle- and wheelchaircontrol methods have been suggested for people with MD, for instance based on brain-computer-interaction $(\mathrm{BCl})[30$, $31,13,2,21]$, speech [33, 34], gaze interaction [5, 39, 20], and $\mathrm{fMRI}$ recording of covert visuospatial attention [1].

Brain-computer interaction [30] may be used by people with severe motor neuron diseases such as Amyotrophic Lateral Sclerosis (ALS), even when they can not move any part of their body - a so-called Locked-In Syndrome. Some of the $\mathrm{BCl}$-methods, however, require substantial training and set-up, since caps and gel-based electrodes can be difficult to apply. Also, information transfer with $\mathrm{BCl}$ systems are quite low; for example [9] reported a throughput of 0.05$1.44 \mathrm{bits} / \mathrm{s}$.
Speech interfaces are popular for mobile phones and smart speakers. They have been suggested for telerobot navigation also. Unfortunately, some people with MD have impaired speech. Low-level direction guiding by speech can be cumbersome as a user may have to repeat the same command multiple times (e.g. left, left, left), or rely on precise timing [33].

Gaze interaction has been used by people with MD for more than twenty years, mainly for communication and pointer-input [16]. Throughput values for gaze have been reported to be 2.55 bits/s [17]. Gaze-controlled navigation of robots was first studied by Tall et al. in 2009 [28]. Since then, gaze tracking technology has become inexpensive and accurate. However, it remains a challenge to design interfaces that only respond to intentions, and not just eye movements. Head input has shown to be more precise and less erroneous than gaze $[17,6]$ but it may also be tiring to use for longer time.

Besides a traditional set-up with monitors, head-mounted displays (HMD) are now being tested for telerobots with a 360 degree camera mounted on the telerobot [5,39] with gaze- or head- movements changing the field of view [10, 5]. Gaze tracking sensors are built into some recent HMD's. The increased availability of eye-tracking equipment makes it feasible to utilize this technology for explicit control tasks with robotics [35]. Hence, we have enabled gaze control of telepresence robots with an HMD in previous studies [39, 38]. The key findings were that after training, able-bodied participants could effectively navigate telerobots by gaze. VR-simulations also showed to be a safe and efficient training tool for gaze navigation [37]. However, like other studies, e.g. [20], we did not involve people with MD. So it is important now to engage people with MD in the design of gaze-controlled telepresence robots and explore future use- 


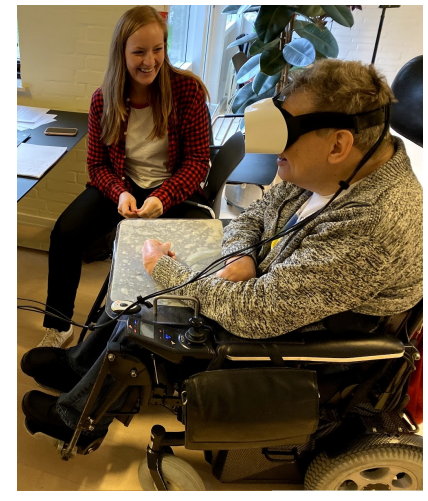

Figure 3: A participant wearing an HMD with a built-in eye tracker.

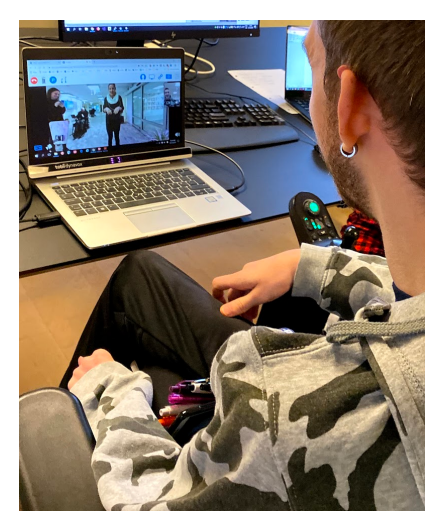

Figure 4: A participant using the Double robot in front of a screen and the Tobii eye trackers. In this case the experimenter was standing behind the user and emulating his gaze movements by use of a joystick, applying a Wizard of $\mathrm{Oz}$ technique. cases with them. We initiated this process with five participants in a field test presented in this paper.

\section{Field study}

Our study applied experience prototyping [3] and the Wizard of Oz technique [24]. The goal was to observe the use and effectiveness of various interfaces and control methods with our target users, rather than to compare a range of complete robotic systems. We therefore used the Wizard of $\mathrm{Oz}$ technique for some of our scenarios because of its advantages in early explorations, where complex system intelligence can be delivered by a human assistant instead of being fully implemented. Also, without an open API to the Double robots it would be a demanding task to establish a direct gaze control.

According to principles suggested by [25] when conducting accessibility research, our study-plan and consent form were sent to the caregivers before the study. Observations and conversations during the study were noted by the experimenter, supported by records of the participants' spontaneous verbalisation, system log files, screen recordings, and room video recordings for post-hoc analysis.

\section{Participants}

Five people (age 21 -55 years; 3 men and 2 women) with different levels of motor impairments participated. They live in a care-home together with 90 people who use wheelchairs for daily mobility. Some of the residents use gaze interaction for communication and some use speech commands for smart-home control. All of our five participants have impaired manual activity, for instance limited gripping, fingering or holding ability, due to cerebellum disorders or cerebral palsy. None of them have cognitive impairments or literacy difficulties, but one of them has impaired speech functions. Three of them have experienced VR using an HMD and one has experienced a telerobot.

\section{Procedure}

Participants conducted the study one by one. After greetings and a brief introduction, we collected demographic information, including experience with gaze control, VR, telerobots and wheelchair control. For the one individual who is non-verbal, this information was provided by a care giver. In particular, participants were informed that if they felt uncomfortable at any time they should stop immediately. Then we showed a demo video of one of our earlier telepresence robot projects [5] with a person controlling a telerobot by his gaze while laying in bed. Two different telerobots (see fig. 2) were standing next to the table we were sitting at.

Initially, we conducted an interview focusing on their expectations and visions of possible usage of telerobots for their daily life activities. Then followed the two experience prototyping sessions as described below. We presented a range of options to them, including types of displays (HMD versus screen), independence levels (independent versus assisted by others), and control methods (gaze, speech or hand).

\section{Gaze navigation}

In the first experience prototyping session, we used our gaze-controlled robotic telepresence system (see Fig. 5). An HMD (FOVE) was connected with a computer running Unity (see Fig. 3). We first gave the participants the possibility to train navigating of a virtual robot in VR by conducting six trials, similar to the procedure used in [37]. When using the VR simulator for training of gaze control, the simulated "live" video stream was generated from the VR engine. The point-of-view and view height were similar to the real telerobot and so were the virtual robot's maneuverability. Both the virtual and the real robot (see next section) were navigated in a room of $4 \times 5 \mathrm{~m}$. 


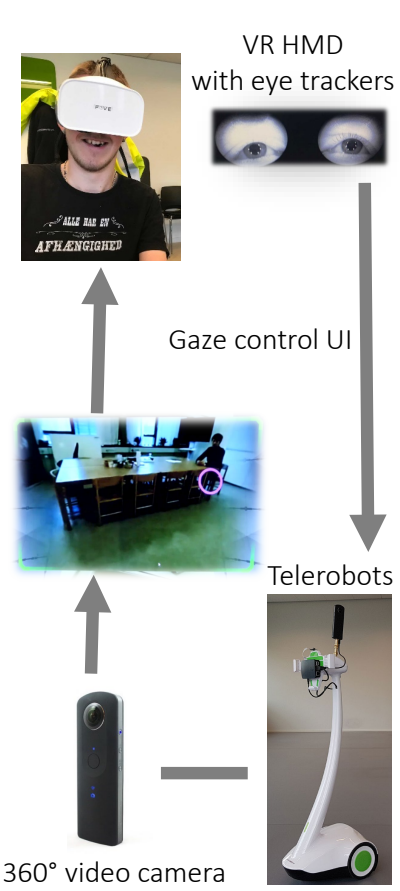

Figure 5: System architecture of our gaze-controlled telepresence robots with an HMD.
Then they got to try a real telerobot. A Padbot with a 360 degree RICOH THETA S camera was controlled via a wireless network through a Raspberry Pi (see Fig. 2). We introduced two ways of driving the telerobots, namely one mode termed "invisible control" [38] and the other termed "way-point control" (see Fig. 6). The way-point control was similar to the control mode found in the Double 3 model, where users click on the floor to point at the next location for the robot to drive. In our case, way-points were marked by dwelling with gaze at the floor for a set dwell time.

Participants were asked to use their gaze to navigate the telerobot, wearing a FOVE HMD with build-in eye tracking. They were instructed to drive around a large table standing in the middle of the room and say hello to a person they would meet while driving around it. They did this twice - once using the "invisible control" which was a direct drive-where-you-look steering principle, and once using the way-point method (see Fig. 6). Afterwards, they answered questions about how confident they felt using gaze control and about their preference for one of the two gaze control methods.

Wizard of $\mathrm{Oz}$ navigation by speech and gaze In the second experience prototyping session, we presented a Double robot (see Fig. 2) running the Double web application in a Chrome browser on a laptop (HP elitebook with a 15" monitor) connected to the robot via wireless network.

Two levels of assistance were experienced by the participants. First they could ask a helper to navigate the robot from the corner of the room to their position. Then we gave the participants an open instruction of..."Your next task is to move the robot outside the door. You can just tell the robot what you would like it to do...." This was done to provide a possibility to imagine the level of intelligence they would ex- pect for this type of interaction. In response, they gave different kinds of commands, for instance, simply "move outside"; or "find the door", and then "go outside"; or just simple direction commands like "left, left, stop". Their speech commands were actually executed by the experimenter standing behind them with a joystick. Participants were not informed that their speech commands were manually performed by the experimenter.

Then they were asked to use gaze control. An eye tracker (Tobii Eye trackers $4 C$ ) was used for tracking the user's gaze point and shown on top of the live video stream from the Double robot (see Fig. 4). Again, steering of the Double was performed in a Wizard of Oz set-up by an experimenter standing behind the participant manually following the users gaze point with a joystick (Xbox 360 controller). The Double robot was driven from the experiment room through a corridor to the canteen, passing the reception of the care-home - a total distance of around $40 \mathrm{~m}$.

We finally asked participants to try driving the telerobot around by simple speech commands - for those 4 participants who could do so. Four types of commands were possible: Left, right, stop and go. The experimenter executed their commands with his joystick behind their back. Three participants also tried to use hand control in the end.

After the sessions, we talked about their experiences and asked questions like: What would you like to use a telerobot for? How would you like to control the robot? How would you like to communicate with people you meet when driving a telerobot?

\section{Observations}

\section{User expectations}

All of our participants assumed the robots would be easy to use before they had tried them, and their initial expectations 


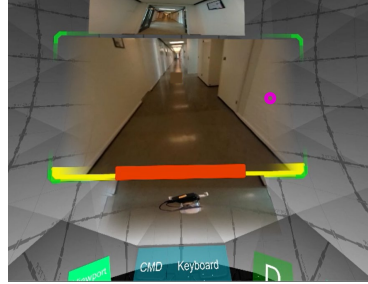

Invisible control

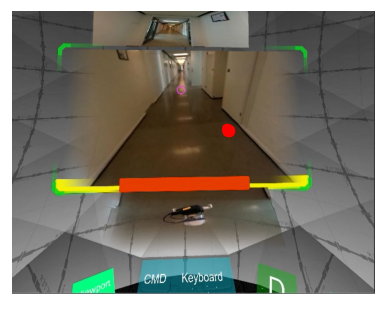

Waypoint control

Figure 6: The two types of gaze control UI: invisible control (top), where the user drives in the direction of sight (indicated by a purple circle), and way-point control (bottom), where the user marks the next way-point by a dwell click on the floor (red circle). were that the telerobots would be fun like a computer game, exciting to drive just with the eyes, a new way of being me. One participant was a bit nervous to use the robots, and asked a helper to hold her hand during the test.

At this early stage, it was difficult for most of them to imagine a use case that they could relate to. Nevertheless, two of them envisioned that they could use telerobots to go shopping by talking with a care giver or a shop assistant via the robot, or just look at the price labels themselves. Once the participants had experienced the telerobots they got a much more clear vision of potential usage, mostly focusing on social interaction and possible inclusion in society. For instance, one participant envisioned that he could get a job in a warehouse driving robot trucks remotely or cleaning floors with a floor cleaning machine. Another participant expressed a wish for the telerobot to have an arm that could do things... Similar applications have been addressed in previous research papers also, for instance, the use of telepresence robots for shopping [36] and for remote work [7].

\section{Control methods}

Autonomous telepresence robots [4] might seem like a good solution for our target users. However, fully-autonomous systems may increase workload if users lack trust in the automated system, especially when the underlying mechanism of automation is not clear to them [19]. None of our users preferred to have the telerobot driven for them, either by other people nor by a fully-autonomous "intelligent" system. This is in line with previous observations [14] that our target users prefer to retain control authority. Therefore, semi-autonomous robots seem to be a more viable solution, using intelligent systems to assist in problematic situations only, and sensors to warn when obstacles are detected. However, this is a very complex issue that requires more research addressing differences in user needs, information transfer rates, differences in robot design, and differences in control principles - to name some of the factors that may impact performance and user experience.

When we asked our participants about their feelings of independence using the categories suggested by [22], (in our terms being able to control robots on your own, being able to maintain personal mobility in a remote place and being confident doing so), all of the participants stated that they had the same feeling of independence when using the different control methods (gaze, speech and hand). Since both the gaze and speech were sometimes controlled by another person in the two Wizard of Oz sessions this is not conclusive. However, when asked which input they liked most, all of the participants except one preferred gaze. In particular, the individual who was unable to give speech commands seemed very excited when using gaze interaction - she made a lot of gestures with a smile on her face. One participant was not able to get a sufficiently good gaze calibration with the HMD. The other four participants quickly gained confidence with this input method when driving in the VR simulator.

We had great expectations for the way-point control method. However, most of our participants found it very difficult to use and one of them did not finish driving around the table. Two of them mentioned that setting way-points forced them to look too far down and prevented them from attending to people and looking around in the room, c.f. Fig. 7. Hence, in future designs, we will focus on better ways to mark a way-point on the floor by gaze.

Hardware

Comparing the Padbot (without a face monitor) and the Double robot, some of the participants stated they preferred that "...people could see you the same way you can see 


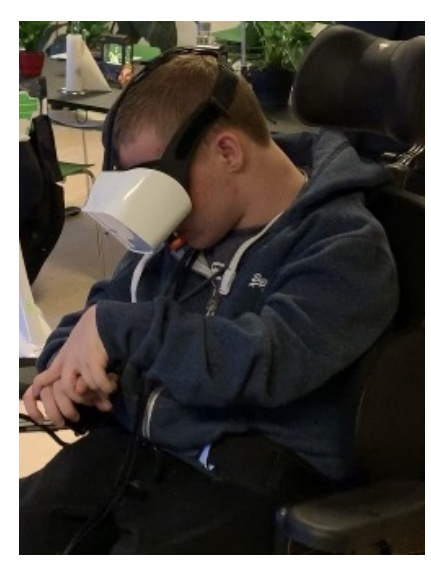

Figure 7: A participant using the way-point control to navigate a telerobot. He commented that this forced him to look too far down and prevented him from attending to people and to see the environment. them" and that the Double is a robot "... where I could be with my family and they could see me and I could see and talk to them. Interestingly, none of them commented on the option of having the 360 degree view provided by the Padbot, and only rarely did they make use of it. Instead of turning their head when wearing a HMD, they simply turned the robot to change their field of view.

HMDs hold potential advantages for telerobot interaction, for instance improved immersion and natural head control of field of view. However, two participants preferred to use the screen, rather than the HMD. One of them explained that he did not like the HMD because it would prevent him from being completely independent, since he would always need assistance to strap it on and adjust its position. Another participant could not achieve a sufficiently good gaze calibration with the HMD but he had no problems to get calibrated with a screen-based eye-tracker. Another important reason to consider a screen-based display instead of HMD's is that it allows users to show their face on the remote screen and thus engage in facial communication. This is not possible when the face is covered by a HMD. Some of these problems may be due to current limitations of HMD's and hopefully better solutions (e.g. smart glasses) will become available in the near future.

Presence and Experience

We frequently asked the drivers where are you now ? and the answers to this question were always their remote position (e.g. in the corridor, reception, or canteen) - not their actual physical location (e.i. in the experiment room). This indicates a strong sense of telepresence. Our final - and maybe strongest - impression from this field study was the excitement we observed among all the participants and among their fellow residents and staff in the care-home (see Fig. 1). A staff member even hugged the telerobot when she saw who was driving it. The participant who could not speak, laughed and used her hand gestures when communicating with friend she met on her way. Overall, the participants' assessment of their experiences with telerobots were very positive, with typical statements like "...super fun, easy to use", and even "proud of myself".

\section{Conclusion}

Based on new insights provided by our target users, we are confident that several additional use-cases may be uncovered, and accessibility of human-telerobot interaction may be improved, if participants get an opportunity to familiarize with this technology and become involved in the design process. Their engagement fully confirmed Liu's point [15] that providing ways to give and not simply receive help is an important way to support social interaction and integration.

\section{Acknowledgements}

Thanks to the people at Jonstrupvang care-home who participated in this study. The research has been supported by the China Scholarship Council and the Bevica Foundation. Thanks to Astrid Kofod Trudslev and Nils David Rasamoel for assisting with the study.

\section{REFERENCES}

[1] Patrik Andersson, Josien PW Pluim, Max A Viergever, and Nick F Ramsey. 2013. Navigation of a telepresence robot via covert visuospatial attention and real-time fMRI. Brain topography 26, 1 (2013), 177-185.

[2] Gloria Beraldo, Morris Antonello, Andrea Cimolato, Emanuele Menegatti, and Luca Tonin. 2018. Brain-Computer Interface meets ROS: A robotic approach to mentally drive telepresence robots. In 2018 IEEE International Conference on Robotics and Automation (ICRA). IEEE, 1-6. 
[3] Marion Buchenau and Jane Fulton Suri. 2000. Experience prototyping. In Proceedings of the 3rd conference on Designing interactive systems: processes, practices, methods, and techniques. 424-433.

[4] Akansel Cosgun, Dinei A Florencio, and Henrik I Christensen. 2013. Autonomous person following for telepresence robots. In 2013 IEEE International Conference on Robotics and Automation. IEEE, 4335-4342.

[5] John Paulin Hansen, Alexandre Alapetite, Martin Thomsen, Zhongyu Wang, Katsumi Minakata, and Guangtao Zhang. 2018. Head and gaze control of a telepresence robot with an HMD. In Proceedings of the 2018 ACM Symposium on Eye Tracking Research \& Applications. Article 82.

[6] John Paulin Hansen, Kristian Tørning, Anders Sewerin Johansen, Kenji Itoh, and Hirotaka Aoki. 2004. Gaze typing compared with input by head and hand. In Proceedings of the 2004 symposium on Eye tracking research \& applications. 131-138.

[7] John Paulin Hansen, Astrid Kofod Trudslev, Sara Amdi Harild, Alexandre Alapetite, and Katsumi Minakata. 2019. Providing access to VR through a wheelchair. In Extended Abstracts of the $2019 \mathrm{CHI}$ Conference on Human Factors in Computing Systems. 1-8.

[8] Yasamin Heshmat, Brennan Jones, Xiaoxuan Xiong, Carman Neustaedter, Anthony Tang, Bernhard E Riecke, and Lillian Yang. 2018. Geocaching with a beam: Shared outdoor activities through a telepresence robot with 360 degree viewing. In Proceedings of the $2018 \mathrm{CHI}$ Conference on Human Factors in Computing Systems. 1-13.
[9] Elisa Mira Holz, Johannes Höhne, Pit Staiger-Sälzer, Michael Tangermann, and Andrea Kübler. 2013.

Brain-computer interface controlled gaming: Evaluation of usability by severely motor restricted end-users. Artificial intelligence in medicine 59, 2 (2013), 111-120.

[10] Dhanraj Jadhav, Parth Shah, and Henil Shah. 2018. A Study to design VI classrooms using Virtual Reality aided Telepresence. In 2018 IEEE 18th International Conference on Advanced Learning Technologies. IEEE, 319-321.

[11] Annica Kristoffersson, Silvia Coradeschi, and Amy Loutfi. 2013. A review of mobile robotic telepresence. Advances in Human-Computer Interaction 2013 (2013).

[12] Min Kyung Lee and Leila Takayama. 2011. "Now, I have a body" uses and social norms for mobile remote presence in the workplace. In Proceedings of the SIGCHI conference on human factors in computing systems. 33-42.

[13] Robert Leeb, Serafeim Perdikis, Luca Tonin, Andrea Biasiucci, Michele Tavella, Marco Creatura, Alberto Molina, Abdul Al-Khodairy, Tom Carlson, and José dR Millán. 2013. Transferring brain-computer interfaces beyond the laboratory: successful application control for motor-disabled users. Artificial intelligence in medicine 59, 2 (2013), 121-132.

[14] Robert Leeb, Luca Tonin, Martin Rohm, Lorenzo Desideri, Tom Carlson, and Jose del R Millan. 2015. Towards independence: a BCl telepresence robot for people with severe motor disabilities. Proc. IEEE 103, 6 (2015), 969-982. 
[15] Peng Liu, Xianghua Ding, and Ning Gu. 2016. "Helping Others Makes Me Happy" Social Interaction and Integration of People with Disabilities. In Proceedings of the 19th ACM Conference on Computer-Supported Cooperative Work \& Social Computing. 1596-1608.

[16] Päivi Majaranta and Andreas Bulling. 2014. Eye tracking and eye-based human-computer interaction. In Advances in physiological computing. Springer, London, 39-65.

[17] Katsumi Minakata, John Paulin Hansen, I Scott MacKenzie, Per Bækgaard, and Vijay Rajanna. 2019. Pointing by gaze, head, and foot in a head-mounted display. In Proceedings of the 11th ACM Symposium on Eye Tracking Research \& Applications. Article 50.

[18] Paul D Nisbet. 2002. Who's intelligent? Wheelchair, driver or both?. In Proceedings of the International Conference on Control Applications, Vol. 2. IEEE, 760-765.

[19] Raja Parasuraman, Thomas B Sheridan, and Christopher D Wickens. 2008. Situation awareness, mental workload, and trust in automation: Viable, empirically supported cognitive engineering constructs. Journal of Cognitive Engineering and Decision Making 2, 2 (2008), 140-160.

[20] Alexey Petrushin, Giacinto Barresi, and Leonardo S Mattos. 2018a. Gaze-controlled Laser Pointer Platform for People with Severe Motor Impairments: Preliminary Test in Telepresence. In 2018 40th Annual International Conference of the IEEE Engineering in Medicine and Biology Society. IEEE, 1813-1816.

[21] Alexey Petrushin, Jacopo Tessadori, Giacinto Barresi, and Leonardo S Mattos. 2018b. Effect of a Click-Like
Feedback on Motor Imagery in EEG-BCl and Eye-Tracking Hybrid Control for Telepresence. In 2018 IEEE/ASME International Conference on Advanced Intelligent Mechatronics (AIM). IEEE, 628-633.

[22] Parvaneh Rabiee. 2013. Exploring the relationships between choice and independence: experiences of disabled and older people. British Journal of Social Work 43, 5 (2013), 872-888.

[23] Irene Rae and Carman Neustaedter. 2017. Robotic telepresence at scale. In Proceedings of the $2017 \mathrm{CHI}$ Conference on Human Factors in Computing Systems. 313-324.

[24] Laurel D Riek. 2012. Wizard of Oz studies in HRI: a systematic review and new reporting guidelines. Journal of Human-Robot Interaction 1, 1 (2012), 119-136.

[25] Dianne Rios, Susan Magasi, Catherine Novak, and Mark Harniss. 2016. Conducting accessible research: including people with disabilities in public health, epidemiological, and outcomes studies. American journal of public health 106, 12 (2016), 2137-2144.

[26] Thomas B Sheridan. 1989. Telerobotics. Automatica 25, 4 (1989), 487-507.

[27] Rachel E Stuck, Jordan Q Hartley, Tracy L Mitzner, Jenay M Beer, and Wendy A Rogers. 2017.

Understanding attitudes of adults aging with mobility impairments toward telepresence robots. In

Proceedings of the Companion of the 2017 ACM/IEEE International Conference on Human-Robot Interaction. 293-294. 
[28] Martin Tall, Alexandre Alapetite, Javier San Agustin, Henrik HT Skovsgaard, John Paulin Hansen,

Dan Witzner Hansen, and Emilie Møllenbach. 2009. Gaze-controlled driving. In CHI'09 Extended Abstracts on Human Factors in Computing Systems. ACM, 4387-4392.

[29] Fumihide Tanaka, Toshimitsu Takahashi, Shizuko Matsuzoe, Nao Tazawa, and Masahiko Morita. 2014. Telepresence robot helps children in communicating with teachers who speak a different language. In Proceedings of the 2014 ACM/IEEE international conference on Human-robot interaction. 399-406.

[30] Luca Tonin, Tom Carlson, Robert Leeb, and José del R Millán. 2011. Brain-controlled telepresence robot by motor-disabled people. In 2011 Annual International Conference of the IEEE Engineering in Medicine and Biology Society. IEEE, 4227-4230.

[31] Luca Tonin, Robert Leeb, Michele Tavella, Serafeim Perdikis, and José del R Millán. 2010. The role of shared-control in $\mathrm{BCl}$-based telepresence. In 2010 IEEE International Conference on Systems, Man and Cybernetics. IEEE, 1462-1466.

[32] Katherine M Tsui, Munjal Desai, Holly A Yanco, and Chris Uhlik. 2011. Exploring use cases for telepresence robots. In 2011 6th ACM/IEEE International Conference on Human-Robot Interaction (HRI). IEEE, 11-18.

[33] Katherine M Tsui, Kelsey Flynn, Amelia McHugh, Holly A Yanco, and David Kontak. 2013. Designing speech-based interfaces for telepresence robots for people with disabilities. In 2013 IEEE 13th International Conference on Rehabilitation Robotics (ICORR). IEEE, 1-8.
[34] Katherine M Tsui, Eric McCann, Amelia McHugh, Mikhail Medvedev, Holly A Yanco, David Kontak, and Jill L Drury. 2014. Towards designing telepresence robot navigation for people with disabilities.

International Journal of Intelligent Computing and Cybernetics 7, 3 (2014), 307.

[35] Ginger S Watson, Yiannis E Papelis, and Katheryn C Hicks. 2016. Simulation-based environment for the eye-tracking control of tele-operated mobile robots. In Proceedings of the Modeling and Simulation of Complexity in Intelligent, Adaptive and Autonomous Systems 2016 (MSCIAAS 2016) and Space Simulation for Planetary Space Exploration (SPACE 2016). 1-7.

[36] Lillian Yang, Carman Neustaedter, and Thecla Schiphorst. 2017. Communicating through a telepresence robot: A study of long distance relationships. In Proceedings of the $2017 \mathrm{CHI}$ Conference Extended Abstracts on Human Factors in Computing Systems. 3027-3033.

[37] Guangtao Zhang and John Paulin Hansen. 2019. A Virtual Reality Simulator for Training Gaze Control of Wheeled Tele-Robots. In 25th ACM Symposium on Virtual Reality Software and Technology. Article 49.

[38] Guangtao Zhang, John Paulin Hansen, and Katsumi Minakata. 2019a. Hand- and gaze-control of telepresence robots. In Proceedings of the 11th ACM Symposium on Eye Tracking Research \& Applications. Article 70.

[39] Guangtao Zhang, John Paulin Hansen, Katsumi Minakata, Alexandre Alapetite, and Zhongyu Wang. 2019b. Eye-gaze-controlled telepresence robots for people with motor disabilities. In 2019 14th ACM/IEEE International Conference on Human-Robot Interaction. IEEE, 574-575. 